\title{
10. OBJECTIVE PRISM SURVEYS FOR NEW WHITE DWARFS
}

\author{
C. B. STEPHENSON \\ Warner and Swasey Observatory, \\ Case Western Reserve University, East Cleveland, O., U.S.A.
}

\begin{abstract}
The spectroscopic criteria for identifying strong-lined DA-type stars on low-dispersion objective prism plates are reviewed, as are the objective prism surveys made to date with discovery of white dwarfs a partial or major objective (Table I). It is claimed that there may be upwards of 100 undiscovered white dwarfs brighter than B magnitude 14.0. Some data for all the white dwarfs so far discovered by objective prism are collected in Table II; new identification charts are given for two of these.
\end{abstract}

The only type of white dwarf capable of secure spectroscopic recognition on objective prism plates of low dispersion (say $\sim 600 \AA / \mathrm{mm}$ ) is the class of DA stars - fortunately, a rather populous class - having strong hydrogen lines. As Figure 1 shows, at such spectral dispersions these stars somewhat resemble normal A-type dwarfs, with the following exceptions: the Balmer lines are deep and very broad, and disappear with $\mathrm{H} \varepsilon$ or $\mathrm{H} \xi$; there is no perceptible Balmer discontinuity; and there are no other lines.

The spectral properties just mentioned are fairly well understood theoretically, and for us the only remaining spectroscopic points of interest are the known sources of possible spectral classification error. These are as follows. First, the weak-lined DA stars may easily be confused with rapidly rotating B stars, unless the rapid rotator betrays itself either by the presence of line or Balmer continuum emission or by a significant Balmer discontinuity. But since the Bnn stars can never have deep lines, they can of course never mimic the strong-lined DA stars. Second, and last, a close overlap of the objective prism spectra of two early A-type or late B-type dwarfs, with slight separation in the direction of spectral dispersion, resembles a DA spectrum in the blue region. However, this is a possible cause of false DA classification only if the plate lacks the ultraviolet.

Granted that safe identification of strong-lined DA stars is possible in the manner outlined, the fact that these plates reach only to magnitude 13 or 14 would seem to make white dwarf discovery by objective prism rather limited in power in comperison, say, with proper motion surveys. Nevertheless about half of the 40-odd DA stars brighter than B magnitude 14.0 were discovered from their colors or objective prism spectra, notwithstanding that the sky has been more thoroughly surveyed for large proper motions to this magnitude limit than for colors or spectral types. It appears from the data of Eggen and Greenstein (1965) that the white dwarfs found in proper motion surveys tend to be high-velocity stars; the mean space motion for such DA stars is nearly $80 \mathrm{~km} / \mathrm{sec}$ and even for those brighter than B magnitude 14.0 it is nearly $50 \mathrm{~km} / \mathrm{sec}$. Nevertheless there are large numbers of low-velocity white dwarfs (cf. Greenstein, 1965a). To magnitude 14 there may well remain more undiscovered white dwarfs than those so far found in proper motion surveys. Indeed the third brightest white dwarf then known was discovered as late as 1967 (Stephenson et al., 


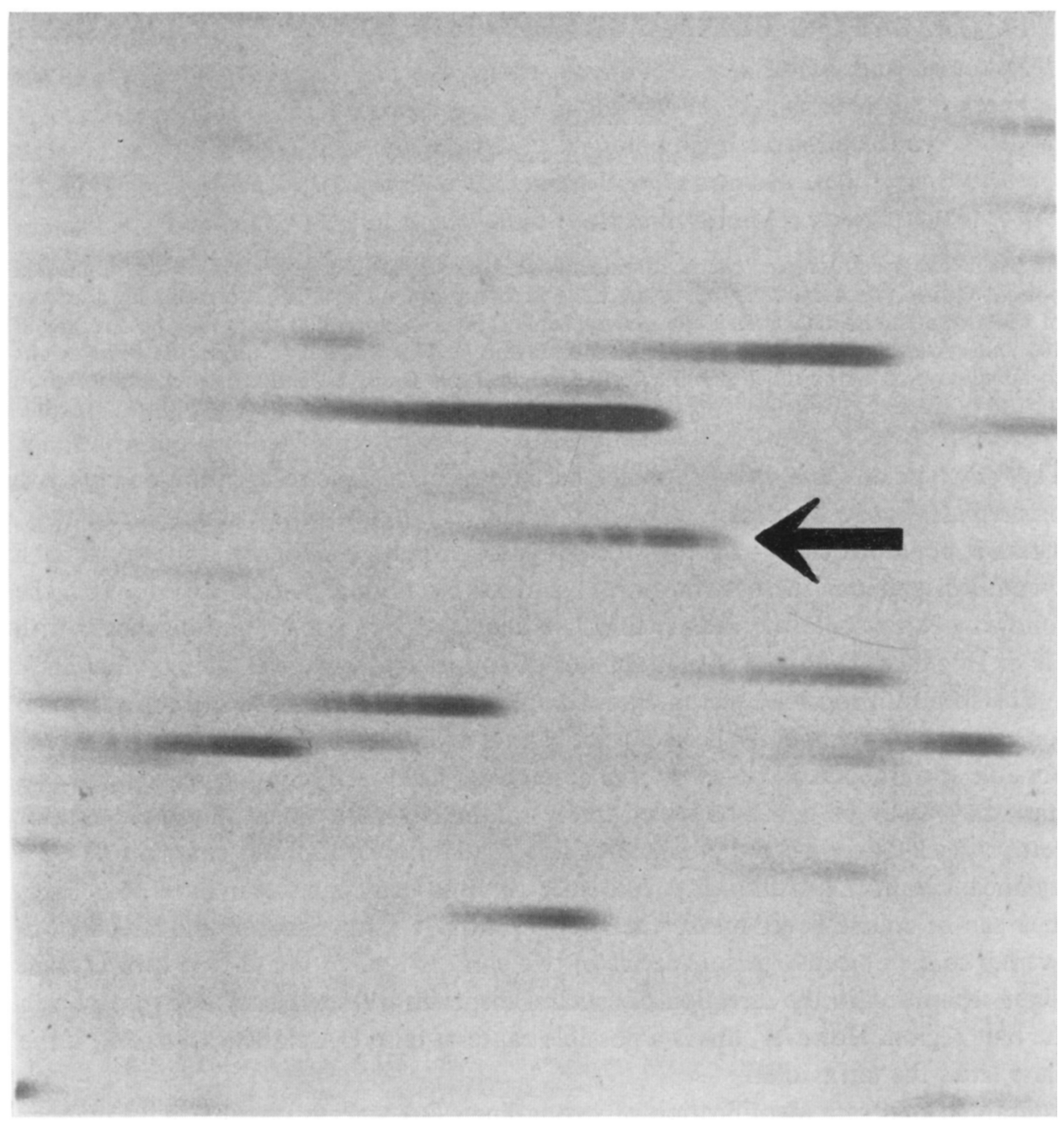

Fig. 1. Portion of a Warner and Swasey Observatory objective prism plate of the field of the DAtype white dwarf Greenwich Astrographic $+73^{\circ} 8031$ (arrow). Original dispersion $580 \AA / \mathrm{mm}$ at $\mathrm{H} \gamma$; red limit $\sim 4900 \AA$, on the right, and blue limit $\sim 3300 \AA$. North is to the left and east below. For comparison, note the B star northeast of the white dwarf. The B magnitude of the white dwarf is 12.9

(Eggen and Greenstein, 1965).

1968) from its objective prism spectrum and another, even brighter though slightly less certain, was similarly discovered a little later (Bidelman, 1968). One may also conclude that there may be a substantial number, say 50 or so, undiscovered bright white dwarfs on the basis of Luyten's discussion of the space density of white dwarfs (Luyten, 1958). This conclusion does not by any means lessen the importance of the vital proper motion surveys of the past and present, but it does make the subject of this paper more than academic. 


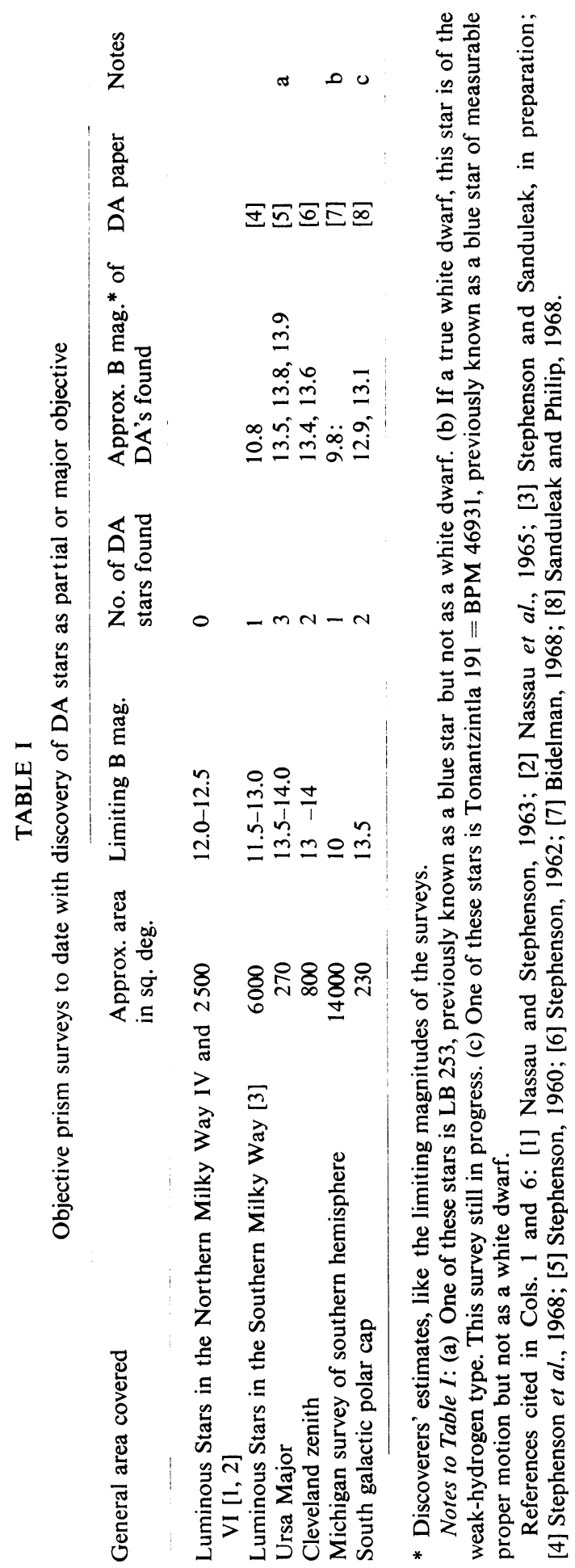




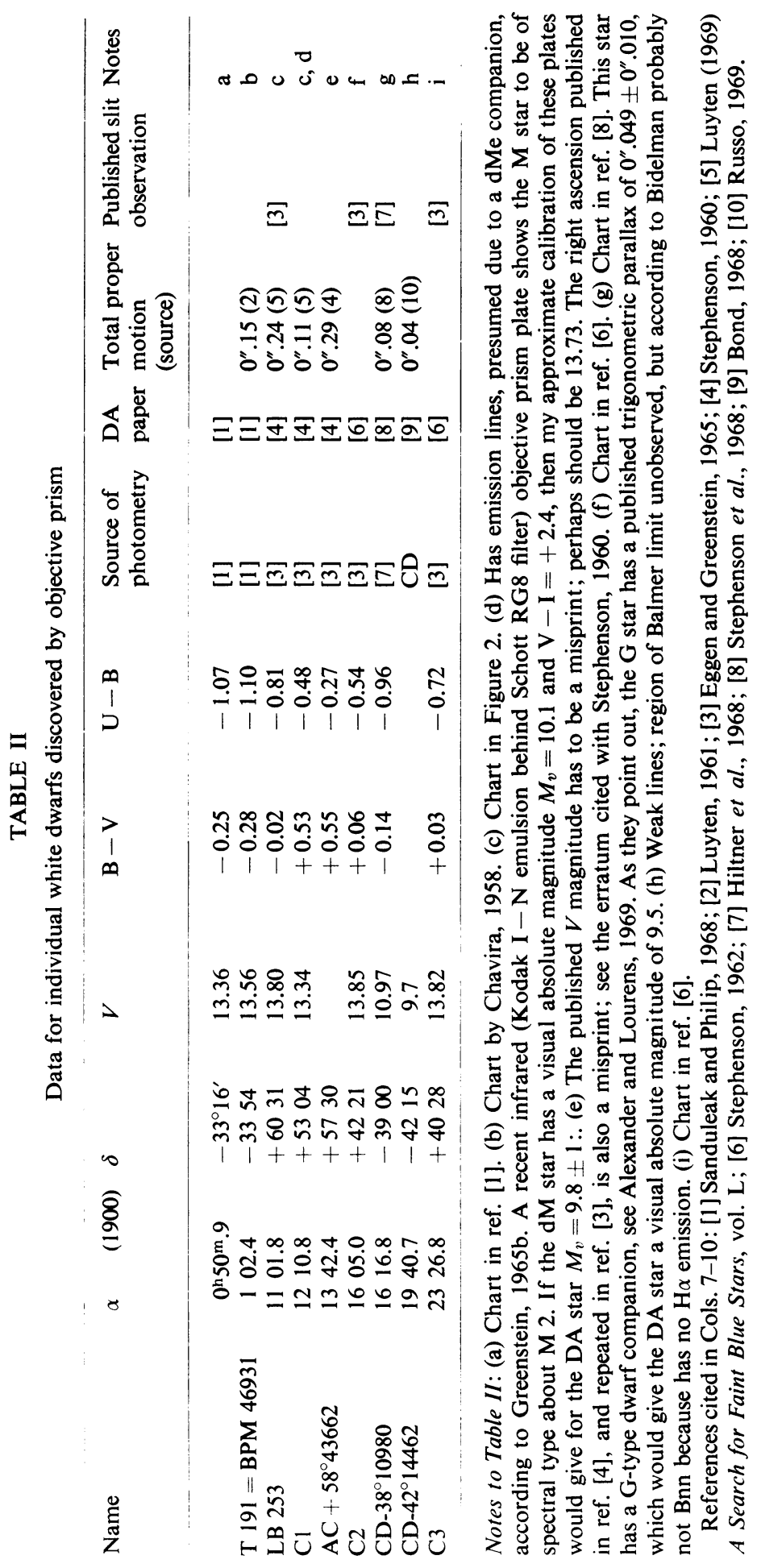




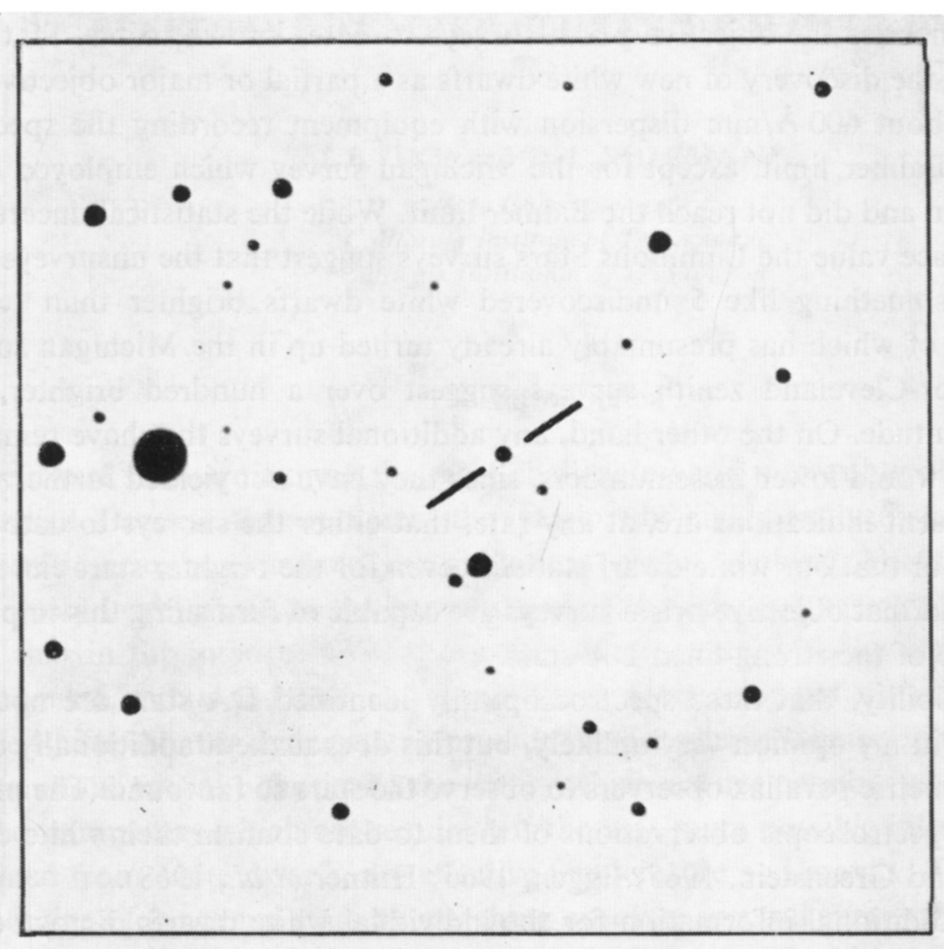

ส

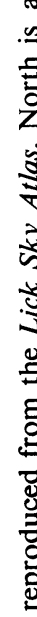

$\cong$

돈

iी

$\stackrel{5}{\pi}$

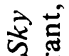

芯㞼

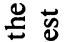

통

잉

异害望

ㅁํㄴ

흉

रิष्ञ

오.

몰

뜽

苛范茨

드응

氠

은

흘 웜

हैं

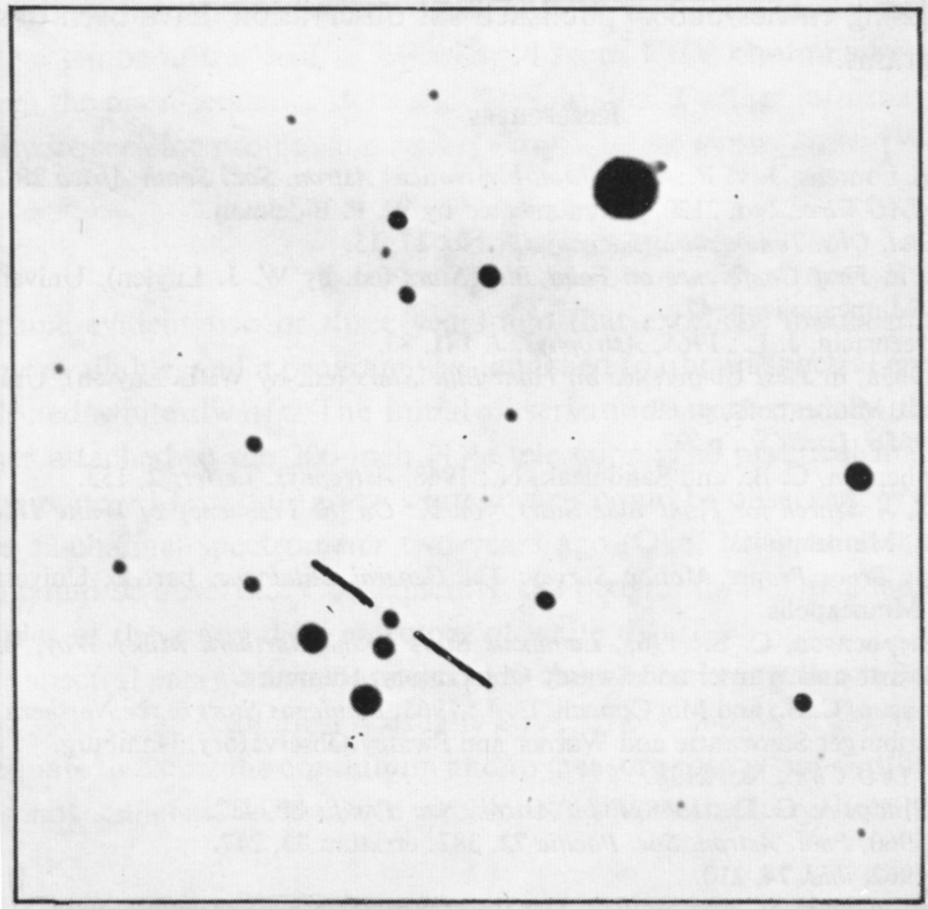


Table I summarizes the objective prism surveys to date, known to me, that have been made with the discovery of new white dwarfs as a partial or major objective. All were done at about $600 \AA / \mathrm{mm}$ dispersion with equipment recording the spectrum well below the Balmer limit, except for the Michigan survey which employed much higher dispersion and did not reach the Balmer limit. While the statistical uncertainty is immense, at face value the Luminous Stars surveys suggest that the unsurveyed sky might contain something like 5 undiscovered white dwarfs brighter than twelfth magnitude, one of which has presumably already turned up in the Michigan survey. The Ursa Major-Cleveland zenith surveys suggest over a hundred brighter than fourteenth magnitude. On the other hand, any additional surveys that have remained unknown to me would lower these numbers, since they have not yielded further white dwarfs. The present indications are, at any rate, that either the surveys to date have been very lucky or that our white dwarf statistics even for the brighter stars can stand improvement and that objective prism surveys are capable of furnishing this improvement in the case of the strong-lined DA stars.

Another possibility, that these spectroscopically identified DA stars are not true white dwarfs, is in my opinion very unlikely, but this does make it additionally desirable for trigonometric parallax observers to observe the stars so far found. The photometric and slit-spectroscopic observations of them to date confirm their white dwarf nature (Eggen and Greenstein, 1965; Eggen, 1965; Hiltner et al., 1968).

Table II list additional information for the individual white dwarfs discovered to date by objective prism. For convenience, some of the data of Table I are repeated here. Stars possessing entries under 'published slit observation' have been observed with slit spectrograms.

\section{References}

Alexander, J. B. and Lourens, J. v. B.: 1969, Monthly Notices Astron. Soc. South Africa $28,95$.

Bond, H. E.: 1968, IAU Circ., No. 2120, communicated by W. P. Bidelman.

Chavira, E.: 1958, Bol. Obs. Tonantzintla Tacubaya 2, No. 17, 15.

Eggen, O. J.: 1965, in First Conference on Faint Blue Stars (ed. by W. J. Luyten), University of Minnesota Press, Minneapolis, p. 47.

Eggen, O. J. and Greenstein, J. L.: 1965, Astrophys. J. 141, 83.

Greenstein, J. L.: 1965a, in First Conference on Faint Blue Stars (ed. by W. L. Luyten), University of Minnesota Press, Minneapolis, p. 62.

Greenstein, J. L.: 1965b, Loc. Cit., p. 97.

Hiltner, W. A., Stephenson, C. B., and Sanduleak, N.: 1968, Astrophys. Letters 2, 153.

Luyten, W. J.: 1958, A Search for Faint Blue Stars, vol. X: On the Frequency of White Dwarfs in Space, Lund Press, Minneapolis.

Luyten, W. J.: 1961, Bruce Proper Motion Survey: The General Catalogue, part E, University of Minnesota Press, Minneapolis.

Nassau, J. J. and Stephenson, C. B.: 1963, Luminous Stars in the Northern Milky Way, vol. IV, Hamburger Sternwarte and Warner and Swasey Observatory, Hamburg.

Nassau, J. J., Stephenson, C. B., and MacConnell, D. J.: 1965, Luminous Stars in the Northern Milky Way, vol. VI, Hamburger Sternwarte and Warner and Swasey Observatory, Hamburg.

Russo, T. W.: 1969, IAU Circ., No. 2128.

Sanduleak, N. and Philip, A. G. D.: 1968, Publ. Astron. Soc. Pacific 80, 437.

Stephenson, C. B.: 1960, Publ. Astron. Soc. Pacific 72, 387; erratum 73, 247.

Stephenson, C. B.: 1962, ibid. 74, 210.

Stephenson, C. B., Sanduleak, N., and Hoffleit, D.: 1968, Publ. Astron. Soc. Pacific 80, 92. 